\title{
Simple Proof for Global Existence of Bohmian Trajectories
}

\author{
Stefan Teufel* and Roderich Tumulka ${ }^{\dagger}$
}

June 15, 2004

\begin{abstract}
We address the question whether Bohmian trajectories exist for all times. Bohmian trajectories are solutions of an ordinary differential equation involving a wavefunction obeying either the Schrödinger or the Dirac equation. Some trajectories may end in finite time, for example by running into a node of the wavefunction, where the law of motion is ill-defined. The aim is to show, under suitable assumptions on the initial wavefunction and the potential, global existence of almost all solutions. We provide a simpler and more transparent proof of the known global existence result for spinless Schrödinger particles and extend the result to particles with spin, to the presence of magnetic fields, and to Dirac wavefunctions. Our main new result are conditions on the current vector field on configuration-space-time which are sufficient for almost-sure global existence.

MSC (2000): $\underline{34 \mathrm{~A} 12}$; 81P99. PACS: 02.30.Hq; 03.65.Ta; 03.65.Pm. Key words: Bohmian mechanics; ordinary differential equations: existence of solutions; equivariant probability distribution; current vector field; Schrödinger equation; Dirac equation.
\end{abstract}

\section{Introduction}

We study a mathematical question arising from and relevant to Bohmian mechanics 15. 1, 11, 3, 10, and its variant based on the Dirac equation 6, 7] (henceforth referred to as the "Bohm-Dirac theory"). In these theories, the motion of particles is defined by ordinary differential equations (ODEs) involving the wavefunction, see (3) and (5) below. The mathematical question we address is global existence, i.e., whether (under what conditions and how often) the particle trajectories are well defined for all times. One obstruction to global existence is that the velocity given by (3) or (15) is singular at the nodes (i.e., zeros) of the wavefunction. In particular, there are trajectories that are not defined for all times because they run into a node. Thus, the strongest statement

\footnotetext{
${ }^{*}$ Mathematics Institute, University of Warwick, CV4 7AL Coventry, UK. E-mail: teufel@maths.warwick.ac.uk

${ }^{\dagger}$ Dipartimento di Fisica dell'Università di Genova and INFN sezione di Genova, Via Dodecaneso 33, 16146 Genova, Italy. E-mail: tumulka@mathematik.uni-muenchen.de
} 
one can expect to be true is that global existence holds for almost all solutions of the equation of motion. As we show, this is in fact true for suitable potentials and initial wavefunctions. As a by-product, one obtains from almost-sure global existence the equivariance of the $\left|\psi_{t}\right|^{2}$ distributions.

The relevance of Bohmian mechanics to the foundations of quantum mechanics arises from the fact that a world governed by Bohmian mechanics satisfies all probability rules of quantum mechanics [5, 1, 11, 3, 10. Bohmian mechanics thus provides an example of a "quantum theory without observers," one in which no reference to observers is needed for the formulation of the theory, and an explanation of the quantum probabilities in terms of objective events.

The authoritative paper on global existence of Bohmian trajectories is by Berndl et al. [4; see also [2. We note that the proof given by Holland [15, p. 85] is incorrect (see 4] for details). We also remark that the general existence theory for first order ODEs with velocity vector fields that are not Lipschitz but only in some Sobolev space 9] does not apply to Bohmian trajectories. The results of 9] hold for vector fields with bounded divergence, while the divergence of a Bohmian velocity field, such as in (3) and (4), typically diverges at nodes of the wave function. Berndl et al. 4] already proved almost-sure global existence for suitable potentials and initial wavefunctions; while they give a proof only for spinless nonrelativistic particles, a similar proof could presumably be devised for Bohmian mechanics with spin [1, 3] and the Bohm-Dirac theory. We provide here an alternative proof that is shorter and more transparent than the proof by Berndl et al. Our result covers all cases covered by their existence theorem; in addition, our result also covers Bohmian mechanics with spin and magnetic fields and Bohm-Dirac theory; for the latter our result and its proof become particularly simple thanks to the fact that the Bohm-Dirac velocities are bounded by the speed of light. Even more generally, our result can be applied to any Bohm-type dynamics, as we formulate conditions on the current vector field on configuration-space-time that are sufficient for almost-sure global existence.

There are three ways in which a trajectory can fail to exist globally: it can approach a node of the wavefunction (where the equation of motion is not defined), it can approach a singularity of the potential (where the equation of motion need not be defined), or it can escape to infinity in finite time. Hence, the main work of any existence proof for Bohm-type dynamics is to show that almost every trajectory avoids the "bad points" (nodes, singularities, infinity) in configuration space. The method of Berndl et al. is based on estimating the probability flux across surfaces surrounding the bad points and pushing these surfaces closer to the bad points; in the limit in which the surfaces reach the bad points, the flux vanishes.

The advantage of our approach is that it does not require skillful estimates and does not involve limits. Instead, our method is based on considering a suitable nonnegative quantity along the trajectory that becomes infinite when the trajectory approaches a bad point; if such a quantity has finite expectation, at least locally, then the set of initial configurations for which it becomes infinite must be a null set. That the expectation be locally finite can be paraphrased as an integral condition on the current vector field.

To illustrate our method, we briefly describe an argument of this kind: the total distance $D$ traveled in configuration space in the time interval $[0, T]$ becomes infinite 
when the trajectory escapes to infinity during $[0, T]$. To prove that $D$ is almost surely finite, we prove that it has finite expectation. A calculation shows that

$$
\mathbb{E} D \leq \int_{0}^{T} d t \int_{\mathbb{R}^{3 N}} d q|J|,
$$

where $J$ is the spatial component of the current vector field. Thus, the finiteness of the right hand side of (11) is a natural condition on the current ensuring that almost no trajectory escapes to infinity in $[0, T]$.

This argument was already sketched in [14; it was inspired by a similar consideration in the global existence proof of [13] for Bell's jump process for lattice quantum field theory, another Markov process depending on a wavefunction $\psi_{t}$ and having distribution $\left|\psi_{t}\right|^{2}$ at any time $t$; the quantity considered there was the number of jumps during $[0, T]$. Finally, a related argument was also described in Remark 3.4.6 of [4], see our Remark 5 for a comparison.

Our method is also in a way more elementary than that of Berndl et al.: we do not make use of the nontrivial fact that the $|\psi|^{2}$-probability of crossing a surface $\Sigma$ in configuration-space-time is bounded by $\int_{\Sigma}|d \sigma \cdot j|$ where $d \sigma$ is the normal on $\Sigma$ with length equal to the area of the surface element and $j$ is the current vector field. Indeed, we use this fact only for surfaces lying in $t=$ const. slices of configuration-space-time, for which it is much simpler to prove, see Lemma 1. To be sure, the statement about general surfaces is interesting in its own right and also relevant to other applications such as scattering theory, but its proof takes several pages in [2].

While our innovation concerns sufficient conditions on the current for almost-sure global existence, there remains the functional analytic question of deriving these conditions from conditions on the potential and the initial wavefunction. We carry this out in several example cases but contribute nothing original; we employ the same arguments as Berndl et al. or standard arguments.

This article is organized as follows. In Section 2 we give the definition of Bohmian trajectories for both the Schrödinger and the Dirac equation; we elucidate the relevance of the current vector field to the trajectories and their distribution. In Section 3 we state and prove our results in terms of a current vector field. In Section 4 we state and prove our results for Bohm-Dirac theory. Finally, in Section 5 we state and prove our results for Bohmian mechanics.

\section{Setup}

We briefly recall Bohmian mechanics and the Bohm-Dirac theory for a system of $N$ particles. Then we describe what singularities we will allow in the potential. Finally, we point out how for both Bohmian mechanics and Bohm-Dirac theory the trajectories arise from a current vector field on configuration-space-time. 


\subsection{Equations of Motion}

In Bohmian mechanics, the wavefunction is a function $\psi: \mathbb{R} \times \mathbb{R}^{3 N} \rightarrow \mathbb{C}^{k}$ where $\mathbb{R}$ represents the time axis, $\mathbb{R}^{3 N}$ the configuration space of $N$ particles, and $\mathbb{C}^{k}$ the value space of the wavefunction representing the internal degrees of freedom of the particles such as spin (and possibly quark flavor etc.). $\psi=\psi\left(t, \boldsymbol{q}_{1}, \ldots, \boldsymbol{q}_{N}\right)$ evolves according to the Schrödinger equation

$$
\mathrm{i} \hbar \frac{\partial \psi}{\partial t}=-\sum_{i=1}^{N} \frac{\hbar^{2}}{2 m_{i}}\left(\nabla_{\boldsymbol{q}_{i}}-\frac{\mathrm{i} e_{i}}{c \hbar} \boldsymbol{A}\left(\boldsymbol{q}_{i}\right)\right)^{2} \psi+V\left(\boldsymbol{q}_{1}, \ldots, \boldsymbol{q}_{N}\right) \psi
$$

where $m_{i}$ and $e_{i}$ denote mass and charge of the $i$-th particle, $c$ the speed of light, $\boldsymbol{A}$ is the external electromagnetic vector potential, and $V$ is the potential, which may be Hermitian $k \times k$-matrix valued. For particles with spin in the presence of magnetic fields, the potential includes a term $\sum_{i} \frac{\hbar e_{i}}{2 m_{i} c}(\nabla \times \boldsymbol{A})\left(\boldsymbol{q}_{i}\right) \cdot \boldsymbol{\sigma}_{i}$ where $\boldsymbol{\sigma}_{i}$ is the vector of spin operators (Pauli matrices for spin- $\frac{1}{2}$ ) acting on the spin index of particle $i$; this form of the Schrödinger equation is known as the Pauli equation.

The law of motion for the trajectory $\boldsymbol{Q}_{i}(t)$ of the $i$-th particle reads

$$
\frac{d \boldsymbol{Q}_{i}}{d t}(t)=\frac{\hbar}{m_{i}} \operatorname{Im} \frac{\psi^{*}\left(\nabla_{\boldsymbol{q}_{i}}-\frac{\mathrm{i} e_{i}}{c \hbar} \boldsymbol{A}\left(\boldsymbol{q}_{i}\right)\right) \psi}{\psi^{*} \psi}(t, Q(t)),
$$

where $Q=\left(\boldsymbol{Q}_{1}, \ldots, \boldsymbol{Q}_{N}\right)$ is the configuration, and $\psi^{*} \phi$ denotes the inner product in $\mathbb{C}^{k}$. The right hand side of (3) is ill-defined when and only when either $\psi(t, Q)=0$ (node of $\psi$ ) or $\psi$ is not differentiable at $(t, Q)$. For an explicit example of a trajectory that runs into a node of $\psi$, see 4 .

In Bohm-Dirac theory, the wavefunction is a function $\psi: \mathbb{R} \times \mathbb{R}^{3 N} \rightarrow \mathbb{C}^{4^{N}}=\left(\mathbb{C}^{4}\right)^{\otimes N}$ evolving according to the Dirac equation

$$
\mathrm{i} \hbar \frac{\partial \psi}{\partial t}=-\sum_{i=1}^{N} \mathrm{ic} \hbar \boldsymbol{\alpha}_{i} \cdot \nabla_{\boldsymbol{q}_{i}} \psi+V\left(\boldsymbol{q}_{1}, \ldots, \boldsymbol{q}_{N}\right) \psi
$$

where $\boldsymbol{\alpha}_{i}$ denotes the vector of Dirac alpha matrices acting on the spin index of particle $i$; we have included the mass terms in the potential $V$, which is Hermitian $4^{N} \times 4^{N}$-matrix valued. In the presence of magnetic fields, $V$ includes a term $-\sum_{i} e_{i} \boldsymbol{A}\left(\boldsymbol{q}_{i}\right) \cdot \boldsymbol{\alpha}_{i}$.

The law of motion for the trajectory $\boldsymbol{Q}_{i}(t)$ of the $i$-th particle reads

$$
\frac{d \boldsymbol{Q}_{i}}{d t}(t)=c \frac{\psi^{*} \boldsymbol{\alpha}_{i} \psi}{\psi^{*} \psi}(t, Q(t))
$$

The right hand side is ill-defined at nodes of $\psi$ and only there.

\subsection{Singularities of the Potential}

Among the physically relevant examples of potentials $V=V\left(\boldsymbol{q}_{1}, \ldots, \boldsymbol{q}_{N}\right)$ is the Coulomb potential,

$$
V\left(\boldsymbol{q}_{1}, \ldots, \boldsymbol{q}_{N}\right)=\sum_{i<j} \frac{e_{i} e_{j}}{\left|\boldsymbol{q}_{i}-\boldsymbol{q}_{j}\right|}
$$


which is singular at coincidence configurations (those with $\boldsymbol{q}_{i}=\boldsymbol{q}_{j}$ for some $i \neq j$ ). This motivates us to allow that $V$ is defined only on a subset $\mathcal{Q} \subset \mathbb{R}^{3 N}$; e.g. in the case of Coulomb interaction, $\mathcal{Q}$ is the set of non-coincidence configurations; in the case of an external Coulomb potential generated by charges located at $\boldsymbol{z}_{1}, \ldots, \boldsymbol{z}_{M}, \mathcal{Q}=$ $\left(\mathbb{R}^{3} \backslash\left\{\boldsymbol{z}_{1}, \ldots, \boldsymbol{z}_{M}\right\}\right)^{N}$. One cannot expect a Schrödinger wavefunction to be differentiable on the singular set $\mathbb{R}^{3 N} \backslash \mathcal{Q}$ of the potential, as exemplified by the ground state of the hydrogen atom, which is proportional to $\exp (-\lambda|\boldsymbol{q}|)$ for suitable $\lambda>0$. Thus, the right hand side of (3) may be ill-defined on $\mathbb{R}^{3 N} \backslash \mathcal{Q}$, and we will use differentiability of $\psi$ only on $\mathcal{Q}$. For the Coulomb interaction and the external Coulomb potential, $\mathcal{Q}$ is of the form $\mathcal{Q}=\mathbb{R}^{d} \backslash \cup_{\ell=1}^{m} S_{\ell}$, where $S_{\ell}$ are hyperplanes. Our method of proof allows somewhat weaker assumptions:

A closed set $S \subset \mathbb{R}^{d}$ is admissible, if there is a $\delta>0$ such that the distance function $q \mapsto \operatorname{dist}(q, S)$ is differentiable on the open set $(S+\delta) \backslash S$, where $S+\delta=\left\{q \in \mathbb{R}^{d}\right.$ : $\operatorname{dist}(q, S)<\delta\}$. Then the configuration space $\mathcal{Q}$ is

$$
\text { either } \mathcal{Q}=\mathbb{R}^{d} \text { or } \mathcal{Q}=\mathbb{R}^{d} \backslash \bigcup_{\ell=1}^{m} S_{\ell},
$$

where $S_{1}, \ldots, S_{m}$ are admissible sets. For example hyperplanes are obviously admissible sets.

\subsection{The Current Vector Field}

There is a common structure behind the laws of motion (3) and (5): they are of the form

$$
\frac{d Q}{d t}(t)=\frac{J(t, Q(t))}{j^{0}(t, Q(t))}
$$

where $j=\left(j^{0}, J\right)$ is the current vector field on configuration-space-time $\mathbb{R} \times \mathcal{Q}$, defined by

$$
j=\left(|\psi|^{2}, \frac{\hbar}{m_{1}} \operatorname{Im} \psi^{*}\left(\nabla_{\boldsymbol{q}_{1}}-\frac{\mathrm{i} e_{1}}{c \hbar} \boldsymbol{A}\left(\boldsymbol{q}_{1}\right)\right) \psi, \ldots, \frac{\hbar}{m_{N}} \operatorname{Im} \psi^{*}\left(\nabla_{\boldsymbol{q}_{N}}-\frac{\mathrm{i} e_{N}}{c \hbar} \boldsymbol{A}\left(\boldsymbol{q}_{N}\right)\right) \psi\right)
$$

in the Schrödinger case and

$$
j=\left(|\psi|^{2}, c \psi^{*} \boldsymbol{\alpha}_{1} \psi, \ldots, c \psi^{*} \boldsymbol{\alpha}_{N} \psi\right)
$$

in the Dirac case. Provided that $\psi$ is sufficiently differentiable, $j$ has the following properties, which we take to be the defining properties of a current vector field:

$$
\begin{aligned}
& j=\left(j^{0}, J\right) \text { is a } C^{1} \text { vector field on } \mathbb{R} \times \mathcal{Q} \\
& \operatorname{div} j=\sum_{\mu=0}^{d} \partial_{\mu} j^{\mu}=0 \\
& j^{0}>0 \text { whenever } j \neq 0 \\
& \int_{\mathcal{Q}} d q j^{0}(t, q)=1 \quad \forall t \in \mathbb{R} .
\end{aligned}
$$


We will call points in $\mathscr{N}=\{(t, q) \in \mathbb{R} \times \mathcal{Q}: j(t, q)=0\}$ the nodes of $j$. We write $\mathscr{N}_{t}=\{q \in \mathcal{Q}: j(t, q)=0\}$ for the set of nodes at time $t$.

Let $Q_{q}(t)$ denote the maximal solution of (8) starting in $q \in \mathcal{Q} \backslash \mathscr{N}_{0}$ defined for $t \in\left(\tau_{q}^{-}, \tau_{q}^{+}\right)$. It is a reparameterization of an integral curve of $j$, see Remark 8 for details. We will formulate our existence theorem first purely in terms of the current vector field, and then apply our result to the currents (9) and (10).

\subsection{Equivariance}

We now explain the notion of equivariance, and what needs to be shown to prove equivariance. We first remark that equivariance is a crucial property of Bohm-type dynamics, in fact the basis of the statistical analysis of Bohmian mechanics [11] and thus the basis of the agreement between the predictions of Bohmian mechanics and the prescriptions of quantum mechanics. We also remark that, while full equivariance will be a consequence of the existence result, a kind of partial equivariance can be obtained before, see Lemma 1 below; our existence proof will exploit this partial equivariance.

Before we define equivariance, we introduce some notation. Let $\mathscr{A}(\mathcal{Q})$ denote the Borel $\sigma$-algebra of $\mathcal{Q}$. Let $\mu_{t}$ be the measure on $\mathscr{A}(\mathcal{Q})$ with density $j^{0}(t)$ relative to Lebesgue measure,

$$
\mu_{t}(B)=\int_{B} d q j^{0}(t, q)
$$

for all $B \in \mathscr{A}(\mathcal{Q})$. By (11d), $\mu_{t}$ is a probability measure; in Bohmian mechanics and Bohm-Dirac theory, $\mu_{t}$ is the $|\psi(t)|^{2}$ distribution. We introduce a formal cemetery configuration $\diamond$ and set $Q_{q}(t):=\diamond$ for all $t \notin\left(\tau_{q}^{-}, \tau_{q}^{+}\right)$, respectively, if $(0, q)$ is a node of $j, Q_{q}(t):=\diamond$ for all $t \neq 0$. Let $\varphi_{t}: \mathcal{Q} \rightarrow \mathcal{Q} \cup\{\diamond\}, \varphi_{t}(q)=Q_{q}(t)$, denote the flow map of (ㅁ) , and let $\varphi: \mathbb{R} \times \mathcal{Q} \rightarrow \mathbb{R} \times(\mathcal{Q} \cup\{\diamond\})$ be the flow map on configuration-space-time defined by $\varphi(t, q)=\left(t, \varphi_{t}(q)\right)$. Let $\mathcal{Q}_{t}=\left\{q \in \mathcal{Q} \backslash \mathscr{N}_{0}: \tau_{q}^{-}<t<\tau_{q}^{+}\right\}=\varphi_{t}^{-1}(\mathcal{Q})$. Standard theorems (see, e.g., Chapter II of [16]) on ODEs imply that $\varphi$ is $C^{1}$ on the maximal domain $\left\{(t, q) \in \mathbb{R} \times\left(\mathcal{Q} \backslash \mathscr{N}_{0}\right): \varphi_{t}(q) \neq \diamond\right\}$, which is open; in particular, also $\mathcal{Q}_{t}$ is an open set.

Let $\rho_{t}$ be the distribution of $Q_{q}(t)$ if $q$ has distribution $\mu_{0}$, i.e.,

$$
\rho_{t}=\mu_{0} \circ \varphi_{t}^{-1}
$$

One says that the family of measures $\mu_{t}$ is equivariant on the time interval $I$ if $\rho_{t}=\mu_{t}$ for all $t \in I$. (The interval $I$ may be finite or infinite.)

Let $\mathscr{I}_{t}:=\varphi_{t}\left(\mathcal{Q}_{t}\right)=\varphi_{t}(\mathcal{Q}) \cap \mathcal{Q}$ be the image of the flow map in $\mathcal{Q}$ at time $t$. The following lemma formulates "partial equivariance."

Lemma 1 Let $j=\left(j^{0}, J\right)$ satisfy (11a), (11b), and (11c). Then for all $B \in \mathscr{A}(\mathcal{Q})$ and all $t \in \mathbb{R}$,

$$
\rho_{t}(B)=\mu_{t}\left(B \cap \mathscr{I}_{t}\right)
$$

We know of two ways of proving this lemma, requiring comparable effort. One proof, given in [4] and in more detail in [2, goes as follows. $\rho_{t}$ has a density that obeys a continuity equation, and $j^{0}$ satisfies the same continuity equation. By uniqueness of 
solutions of this linear partial differential equation, one obtains that $j^{0}(t)$ coincides with the density of $\rho_{t}$ on $\mathscr{I}_{t}$. An alternative proof, which we give below, is based on applying the Ostrogradski-Gauss integral formula to $j$ on a cylinder formed by the trajectories over a polyhedron in $\mathcal{Q}$.

Proof of Lemma 1. Without loss of generality, $t>0$. For any $d$-chain of singular simplices $E$ in $\mathcal{Q}_{t}$, the cylinder $F$ formed by the trajectories over $E, F=\varphi([0, t] \times E)$, is a $d+1$-chain in configuration-space-time $\mathbb{R} \times \mathcal{Q}$. Applying the Ostrogradski-Gauss integral formula to $j$ and $F$, we obtain

$$
0 \stackrel{\text { 111b }}{=} \int_{F} d t d q \operatorname{div} j=\int_{\partial F} d \sigma \cdot j
$$

where $d \sigma$ is the outward pointing surface normal with length $|d \sigma|$ equal to the area of the surface element. The surface $\partial F$ of the cylinder consists of three parts: the mantle $\varphi([0, t] \times \partial E)$, the lid $\varphi(\{t\} \times E)$, and the bottom $\varphi(\{0\} \times E)$. The integral over the mantle vanishes as the mantle consists of integral curves of $j$ and is thus tangent to $j$. The integral over the lid is $\int_{\varphi_{t}(E)} d q j^{0}(t, q)$ and that over the bottom is $-\int_{E} d q j^{0}(0, q)$. Therefore, we obtain

$$
0=\mu_{t}\left(\varphi_{t}(E)\right)-\rho_{0}(E)=\mu_{t}\left(\varphi_{t}(E)\right)-\rho_{t}\left(\varphi_{t}(E)\right) .
$$

Any two measures that agree on the $d$-chains (and thus in particular on the compact rectangles) agree on a $\cap$-stable generator of the $\sigma$-algebra $\mathscr{A}\left(\mathcal{Q}_{t}\right)$ and are, by a standard theorem, equal. Since $\varphi_{t}$ is a bijection $\mathcal{Q}_{t} \rightarrow \mathscr{I}_{t}$, we obtain (14).

What remains to be shown to prove equivariance is that $\mu_{t}\left(\mathcal{Q} \backslash \mathscr{I}_{t}\right)=0$.

\section{A General Existence Theorem}

Let $\mathscr{B}(\mathcal{Q})$ denote the set of all bounded Borel sets in $\mathcal{Q}$.

Theorem 1 Let $\mathcal{Q} \subset \mathbb{R}^{d}$ be a configuration space as defined in (7) and let $j=\left(j^{0}, J\right)$ be a current as defined in (11). Let $T>0$ and let $\varphi_{t}: \mathcal{Q} \rightarrow \mathcal{Q} \cup\{\diamond\}$ denote the flow map of (8). Suppose that

$$
\begin{gathered}
\forall B \in \mathscr{B}(\mathcal{Q}): \quad \int_{0}^{T} d t \int_{\varphi_{t}(B) \backslash\{\diamond\}} d q\left|\left(\frac{\partial}{\partial t}+\frac{J}{j^{0}} \cdot \nabla_{q}\right) j^{0}(t, q)\right|<\infty, \\
\forall B \in \mathscr{B}(\mathcal{Q}): \int_{0}^{T} d t \int_{\varphi_{t}(B) \backslash\{\diamond\}} d q\left|J(t, q) \cdot \frac{q}{|q|}\right|<\infty,
\end{gathered}
$$

and, if $\mathcal{Q}=\mathbb{R}^{d} \backslash \cup_{\ell} S_{\ell}$, in addition that for every $\ell \in\{1, \ldots, m\}$,

$$
\exists \delta>0 \forall B \in \mathscr{B}(\mathcal{Q}): \quad \int_{0}^{T} d t \int_{\varphi_{t}(B) \backslash\{\diamond\}} d q \mathbf{1}\left(q \in\left(S_{\ell}+\delta\right)\right) \frac{\left|J(t, q) \cdot e_{\ell}(q)\right|}{\operatorname{dist}\left(q, S_{\ell}\right)}<\infty .
$$


Here $\operatorname{dist}\left(q, S_{\ell}\right)$ is the Euclidean distance of $q$ from $S_{\ell}$ and $e_{\ell}(q)=-\nabla_{q} \operatorname{dist}\left(q, S_{\ell}\right)$ is the radial unit vector towards $S_{\ell}$ at $q \in \mathcal{Q}$. Recall that for $\delta$ sufficiently small the distance function is differentiable on $S_{\ell}+\delta$.

Then for almost every $q \in \mathcal{Q}$ relative to the measure $\mu_{0}(d q)=j^{0}(0, q) d q$, the solution of (8) starting at $Q(0)=q$ exists at least up to time $T$, and the family of measures $\mu_{t}(d q)=j^{0}(t, q) d q$ is equivariant on $[0, T]$. In particular, if (15), (16) and, if appropriate, (17) are true for every $T>0$, then for $\mu_{0}$-almost every $q \in \mathcal{Q}$ the solution of (8) starting at $q$ exists for all times $t \geq 0$.

\section{Remarks:}

1. We can formulate the meaning of each of the conditions (15), (16), and (17) as follows. If (15) holds, then $\mu_{0}$-almost no trajectory approaches a node during $[0, T]$. If (16) holds, then $\mu_{0}$-almost no trajectory escapes to $\infty$ during $[0, T]$. If (17) holds, then $\mu_{0}$-almost no trajectory approaches a point in the singular set $\cup_{\ell=1}^{m} S_{\ell}$ during $[0, T]$.

2. To obtain existence also for negative times, one can apply Theorem 1 to the time reversed current

$$
\bar{\jmath}(t, q)=\left(j^{0}(-t, q),-J(-t, q)\right) .
$$

The integral curves of $\bar{\jmath}$ are the time reverses of the integral curves of $j$. Obviously, with $j$ also $\bar{\jmath}$ satisfies (11). If $\bar{\jmath}$ satisfies (15), (16), and, if appropriate, (17) for $T>0$, we obtain almost-sure global existence of $Q_{q}(t)$ on $[-T, 0]$.

3. It suffices to consider in (15), (16) and (17) for the sets $B$ instead of all bounded Borel sets just the balls around the origin. This is because enlarging $B$ cannot shrink the integral. For the same reason, it suffices to integrate over $\mathcal{Q} \backslash \mathscr{N}_{t}$ instead of the not easily accessible sets $\varphi_{t}(B) \backslash\{\diamond\}$.

4. Actually the proof of Theorem 1 works in the same way with the following slightly weaker conditions. Instead of (16) it suffices to assume that

$$
\forall B \in \mathscr{B}(\mathcal{Q}) \exists R<\infty: \quad \int_{0}^{T} d t \int_{\varphi_{t}(B) \backslash\{\diamond\}} d q \mathbf{1}(|q|>R)\left|J(t, q) \cdot \frac{q}{|q|}\right|<\infty
$$

and (17) can be replaced by

$$
\forall B \in \mathscr{B}(\mathcal{Q}) \exists \delta>0: \quad \int_{0}^{T} d t \int_{\varphi_{t}(B) \backslash\{\diamond\}} d q \mathbf{1}\left(q \in\left(S_{\ell}+\delta\right)\right) \frac{\left|J(t, q) \cdot e_{\ell}(q)\right|}{\operatorname{dist}\left(q, S_{\ell}\right)}<\infty .
$$

We chose to state the theorem with the stronger assumption to simplify the presentation and because the weaker assumptions will not be used in the following.

Proof of Theorem 11. Let $Q_{q}(t)$ be the maximal solution of (8) starting in $q$, as described in Section 2.3. Since we deal only with positive times in the following, we write $\tau_{q}$ for $\tau_{q}^{+}$ 
There are three ways in which $Q_{q}(t)$ can fail to exist globally: the trajectory can approach a node, approach a point on the singular set $\cup S_{\ell}$, or escape to infinity in finite time. More precisely, if $q \notin \mathscr{N}_{0}$ and $\tau_{q}<\infty$ there exists an increasing sequence $\left(t_{n}\right)_{n \in \mathbb{N}}$ with $t_{n} \rightarrow \tau_{q}$ such that either there is $x \in \mathscr{N}_{\tau_{q}} \cup \bigcup_{\ell=1}^{m} S_{\ell}$ with $Q_{q}\left(t_{n}\right) \rightarrow x$ or $\left|Q_{q}\left(t_{n}\right)\right| \rightarrow \infty$.

To see this, suppose that $\tau_{q}<\infty$ and that such a sequence did not exist. Then $Q_{q}:=\left\{\left(t, Q_{q}(t)\right): t \in\left(0, \tau_{q}\right)\right\} \subset(\mathbb{R} \times \mathcal{Q}) \backslash \mathscr{N}$ would remain bounded and bounded away from the complement $\left(\mathbb{R} \times \cup S_{\ell}\right) \cup \mathscr{N}$. Since $(\mathbb{R} \times \mathcal{Q}) \backslash \mathscr{N}$ is open, there would be a compact set $K \subset(\mathbb{R} \times \mathcal{Q}) \backslash \mathscr{N}$ such that $Q_{q} \subset K^{\circ}$, with $K^{\circ}$ the interior of $K$. However, the vector field $\left(1, J / j^{0}\right)$ is $C^{1}$ on $(\mathbb{R} \times \mathcal{Q}) \backslash \mathscr{N}$ and thus uniformly Lipschitz on $K$. Therefore, all of its maximal integral curves either exist for all times or hit the boundary of $K$, in contradiction to the hypotheses.

Let now $q \notin \mathscr{N}_{0}$ and $\tau_{q} \leq T$. If there is $x \in \mathscr{N}_{\tau_{q}}$ and $\left(t_{n}\right)$ such that $Q_{q}\left(t_{n}\right) \rightarrow x$, then $j^{0}\left(t_{n}, Q_{q}\left(t_{n}\right)\right) \rightarrow 0$. Hence, the total variation of $t \mapsto \log j^{0}\left(t, Q_{q}(t)\right)$ up to time $T$ diverges, i.e., $L_{q}=\infty$ where

$$
L_{q}=\int_{0}^{\min \left(T, \tau_{q}\right)} d t\left|\frac{d}{d t} \log j^{0}\left(t, Q_{q}(t)\right)\right| \quad \text { for } q \in \mathcal{Q} \backslash \mathscr{N}_{0} .
$$

We now show that $L_{\infty}:=\left\{q \in \mathcal{Q} \backslash \mathscr{N}_{0}: L_{q}=\infty\right\}$ is a $\mu_{0}$-null set. For this it suffices to show that for any bounded set $B \in \mathscr{B}(\mathcal{Q}), B \cap L_{\infty}$ is a $\mu_{0}$-null set. For this in turn, it suffices that the average of $L_{q}$ over $B$ relative to the measure $\mu_{0}$ be finite:

$$
\int_{B} d q j^{0}(0, q) L_{q}=\int_{B} d q j^{0}(0, q) \int_{0}^{\min \left(T, \tau_{q}\right)} d t \frac{\left|\frac{d}{d t} j^{0}\left(t, Q_{q}(t)\right)\right|}{j^{0}\left(t, Q_{q}(t)\right)}=
$$

[the order of integration can be changed since the integrand is nonnegative]

$$
\begin{aligned}
& =\int_{0}^{T} d t \int_{B} d q j^{0}(0, q) \mathbf{1}\left(\tau_{q}>t\right) \frac{\left|\frac{d}{d t} j^{0}\left(t, Q_{q}(t)\right)\right|}{j^{0}\left(t, Q_{q}(t)\right)} \\
& =\int_{0}^{T} d t \int_{\varphi_{t}(B) \backslash\{\diamond\}} \rho_{t}\left(d q^{\prime}\right) \frac{\left|\left(\partial / \partial t+\frac{J}{j^{0}} \cdot \nabla_{q^{\prime}}\right) j^{0}\left(t, q^{\prime}\right)\right|}{j^{0}\left(t, q^{\prime}\right)}=
\end{aligned}
$$

[by Lemma 1]

$$
=\int_{0}^{T} d t \int_{\varphi_{t}(B) \backslash\{\diamond\}} d q^{\prime}\left|\left(\partial / \partial t+\frac{J}{j^{0}} \cdot \nabla_{q^{\prime}}\right) j^{0}\left(t, q^{\prime}\right)\right| \stackrel{(15)}{<} \infty .
$$

This shows $\mu_{0}\left(L_{\infty}\right)=0$ and thus that the solution $Q_{q}(t)$ of (8) $\mu_{0}$-almost surely does not approach a node during $[0, T]$.

Now we consider the cases that either

$$
\lim _{n \rightarrow \infty}\left|Q_{q}\left(t_{n}\right)\right|=\infty
$$


or

$$
\exists x \in \cup_{\ell=1}^{m} S_{\ell}: \quad Q_{q}\left(t_{n}\right) \rightarrow x .
$$

Hence, for such initial conditions either the total variation of $t \mapsto\left|Q_{q}(t)\right|$ is infinite, i.e., $D_{q}=\infty$ where

$$
D_{q}=\int_{0}^{\min \left(T, \tau_{q}\right)} d t\left|\frac{d}{d t}\right| Q_{q}(t)|| \quad \text { for } q \in \mathcal{Q} \backslash \mathscr{N}_{0},
$$

or the total variation of $t \mapsto \log \operatorname{dist}\left(Q_{q}(t), S_{\ell}\right)$ restricted to $S_{\ell}+\delta$ is infinite for some $\ell \in\{1, \ldots, m\}$ and any $\delta>0$, in particular for the one in (17), i.e., $V_{q, \ell}=\infty$ where

$$
V_{q, \ell}=\int_{0}^{\min \left(T, \tau_{q}\right)} d t \mathbf{1}\left(Q_{q}(t) \in\left(S_{\ell}+\delta\right)\right)\left|\frac{d}{d t} \log \operatorname{dist}\left(Q_{q}(t), S_{\ell}\right)\right| \quad \text { for } q \in \mathcal{Q} \backslash \mathscr{N}_{0}
$$

Therefore it suffices to show that $D_{\infty}:=\left\{q \in \mathcal{Q} \backslash \mathscr{N}_{0}: D_{q}=\infty\right\}$ and $V_{\infty, \ell}:=\{q \in$ $\left.\mathcal{Q} \backslash \mathscr{N}_{0}: V_{q, \ell}=\infty\right\}$ are $\mu_{0}$-null sets, and for this we proceed as for $L_{\infty}$.

Let $B \in \mathscr{B}(\mathcal{Q})$. Then (8) , followed by exactly the same arguments as for $L_{q}$, shows that local expectations of $D_{q}$ are finite, i.e.

$$
\int_{B} d q j^{0}(0, q) D_{q}=\int_{0}^{T} d t \int_{\varphi t(B) \backslash\{\diamond\}} d q^{\prime}\left|J\left(t, q^{\prime}\right) \cdot \frac{q^{\prime}}{\left|q^{\prime}\right|}\right| \stackrel{[16)}{<} \infty .
$$

Hence $\mu_{0}\left(D_{\infty}\right)=0$. For local expectations of $V_{q, \ell}$ we obtain, again with (8) and Lemma 1

$$
\begin{aligned}
\int_{B} d q j^{0}(0, q) V_{q, \ell} & =\int_{B} d q j^{0}(0, q) \int_{0}^{\min \left(T, \tau_{q}\right)} d t \mathbf{1}\left(Q_{q}(t) \in\left(S_{\ell}+\delta\right)\right)\left|\frac{\dot{Q}_{q}(t) \cdot e_{\ell}\left(Q_{q}(t)\right)}{\operatorname{dist}\left(Q_{q}(t), S_{\ell}\right)}\right| \\
& =\int_{0}^{T} d t \int_{\varphi_{t}(B) \backslash\{\diamond\}} d q^{\prime} \mathbf{1}\left(q^{\prime} \in\left(S_{\ell}+\delta\right)\right)\left|\frac{J\left(t, q^{\prime}\right) \cdot e_{\ell}\left(q^{\prime}\right)}{\operatorname{dist}\left(q^{\prime}, S_{\ell}\right)}\right| \stackrel{\frac{17}{<} \infty .}{<} \infty .
\end{aligned}
$$

Hence also $\mu_{0}\left(V_{\infty, \ell}\right)=0$, concluding the existence part of the theorem.

It remains to show equivariance. Since the probability of reaching $\diamond$ before time $T$ vanishes, we have $\rho_{t}(\mathcal{Q})=1$ for all $t \in[0, T]$. Since $\rho_{t} \leq \mu_{t}$ by Lemma 1 and $\mu_{t}(\mathcal{Q})=1$ by (11d), we must have $\rho_{t}=\mu_{t}$, which is equivariance.

\section{Remarks.}

5. A reasoning closely related to our method of proof is also applied in [4, Remark 3.4.6. There, an expression analogous to (15) is used to control the probability of reaching an $\varepsilon$-neighborhood of $\mathscr{N}$ before letting $\varepsilon \rightarrow 0$. Apart from the fact that the argument is applied there only to the nodes and not to singularities and infinity, it is also unnecessarily complicated, mainly because it considers an $\varepsilon$-neighborhood instead of fully exploiting the integral (19). 
6. The proof of equivariance was the only place where we used the property (11d) of a current vector field. The existence statement of Theorem 1 holds as well if $j$ satisfies (11) except for (11d); in particular, we may allow $\mu_{t}(\mathcal{Q})=\infty$.

7. Here is another equivariance result that does not use (11d): Let $\mathcal{Q}$ be a configuration space as in (17) and let $j$ satisfy (11) except for (11d). Suppose that almost-sure global existence holds in both time directions, starting from any time. Then the family of measures $\mu_{t}$ is equivariant on $\mathbb{R}$.

To see this, note that for equivariance we need to show merely that $\mathcal{Q} \backslash \mathscr{I}_{t}$ is a $\mu_{t}$-null set, or, in other words, that for $\mu_{t}$-almost every $q \in \mathcal{Q}$ the integral curve of $j$ starting in $(t, q)$ reaches back in time to time 0 . But this is immediate from almost-sure global existence in the other time direction, starting at time $t$.

Thus, if both $j$ and $\bar{\jmath}$ as defined in (18) and their time translates satisfy (15), (16), and, if appropriate, (17) for all $T>0$, we obtain equivariance without (11d).

8. Condition (16) can be replaced by the condition

$$
\text { the first order derivatives of } J \text { are bounded on }[0, T] \times \mathcal{Q} \text {. }
$$

To show this, we show that under this assumption every unbounded solution $Q_{q}(t)$ with $\tau_{q} \leq T$ has $L_{q}=\infty$, with $L_{q}$ defined in (19).

To see this, first note that the solutions of (8) are reparameterizations of the integral curves of $j$. In more detail, let $\gamma_{q}(s)=\left(\gamma_{q}^{0}(s), \Gamma_{q}(s)\right)$ be the unique maximal integral curve to $j$,

$$
\frac{d \gamma_{q}(s)}{d s}=j\left(\gamma_{q}(s)\right)
$$

starting in $(0, q) \in \mathbb{R} \times\left(\mathcal{Q} \backslash \mathscr{N}_{0}\right)$ and defined for $s \in\left(\sigma_{q}^{-}, \sigma_{q}^{+}\right)$. Since $j^{0}>0$ outside nodes, $\gamma_{q}^{0}(s)$ is monotonically increasing, and hence the map

$$
s \mapsto t_{q}(s)=\gamma_{q}^{0}(s)=\int_{0}^{s} d \tilde{s} \frac{d \gamma_{q}^{0}}{d \tilde{s}}=\int_{0}^{s} d \tilde{s} j^{0}\left(\gamma_{q}(\tilde{s})\right)
$$

is invertible on its image $\left(\tau_{q}^{-}, \tau_{q}^{+}\right)$, where $\tau_{q}^{ \pm}=\lim _{s \rightarrow \sigma_{q}^{ \pm}} t_{q}(s)$, with inverse $s_{q}(t)$. Since

$$
\frac{d}{d t} \Gamma_{q}\left(s_{q}(t)\right)=\frac{J\left(\gamma\left(s_{q}(t)\right)\right)}{j^{0}\left(\gamma\left(s_{q}(t)\right)\right)}
$$

$Q_{q}(t)=\Gamma_{q}\left(s_{q}(t)\right)$ is the unique maximal solution of (8) with $Q_{q}(0)=q$; it is defined for $t \in\left(\tau_{q}^{-}, \tau_{q}^{+}\right)$.

Now suppose that $\left|Q_{q}\left(t_{n}\right)\right| \rightarrow \infty$ for some $t_{n} \rightarrow \tau_{q}^{+}$. Then also $\left|\Gamma_{q}\left(s_{q}\left(t_{n}\right)\right)\right| \rightarrow \infty$. Since the derivatives of $J$ are bounded, there are constants $A, R>0$ such that $|J(t, q)| \leq A|q|$ for all $t \in[0, T]$ and all $q \in \mathcal{Q}$ with $|q|>R$. Since $d \Gamma_{q} / d s=$ $J\left(\gamma_{q}(s)\right)$, it follows that $\left|\Gamma_{q}(s)\right| \leq \max \left(\left|\Gamma_{q}(0)\right|, R\right) \mathrm{e}^{A s}$; thus, an integral curve of $j$ 
cannot escape to spatial infinity in a finite interval of the parameter $s$; in other words, $\sigma_{q}^{+}=\infty$. But then

$$
\tau_{q}^{+}=\int_{0}^{\infty} d s j^{0}\left(\gamma_{q}(s)\right)<\infty
$$

implies the existence of an increasing sequence $\left(s_{n}\right)$ with $s_{n} \rightarrow \infty$ such that $j^{0}\left(\gamma_{q}\left(s_{n}\right)\right) \rightarrow 0$, and therefore $L_{q}=\infty$.

\section{Global Existence of Bohm-Dirac Theory}

The Dirac Hamiltonian for $N$ particles is

$$
H_{\mathrm{D}}=-\sum_{i=1}^{N} \mathrm{i} c \hbar \boldsymbol{\alpha}_{i} \cdot \nabla_{\boldsymbol{q}_{i}}+V\left(\boldsymbol{q}_{1}, \ldots, \boldsymbol{q}_{N}\right),
$$

where we assume a nonsingular $V \in C^{\infty}\left(\mathbb{R}^{3 N}\right.$, Herm $\left.\left(\mathbb{C}^{4^{N}}\right)\right)$. According to [8], $H_{\mathrm{D}}$ is essentially self-adjoint on $C_{0}^{\infty}\left(\mathbb{R}^{3 N}, \mathbb{C}^{4^{N}}\right)$ and we denote by $H_{\mathrm{D}}$ the unique self-adjoint extension.

Since the Dirac matrices $\boldsymbol{\alpha}$ have eigenvalues \pm 1 , the velocities in (5) are bounded by $c$. Consequently, the Dirac current (10) satisfies $|J| \leq c \sqrt{N} j^{0}$. This fact makes the proof of global existence particularly simple, as expressed in the following corollary to Theorem [1.

Corollary 1 Let $\mathcal{Q}=\mathbb{R}^{d}$ and let $j=\left(j^{0}, J\right)$ be a current as defined in (11). Suppose that there is a global bound on velocities, i.e., a constant $c>0$ such that $|J| \leq c j^{0}$.

Then for $\mu_{0}$-almost all $q \in \mathbb{R}^{d}$, the solution of (8) starting at $Q(0)=q$ exists for all times, and the family of measures $\mu_{t}$ is equivariant.

Proof of Corollary 1. We show that assumptions (15) and (16) of Theorem 1 are satisfied for any $T>0$. The key observation is that due to the bound on velocities, bounded sets in configuration space stay bounded under the flow. More explicitly, for any bounded set $B \in \mathscr{B}\left(\mathbb{R}^{d}\right)$ contained in, say, the ball $B_{r}$ of radius $r>0$ around the origin, $\varphi_{t}(B) \backslash\{\diamond\}$ will be contained in $B_{r+c t}$ and thus in $B_{r+c T}$ provided $t \in[0, T]$. Now $\left|\left(\partial_{t}+\frac{J}{j^{0}} \cdot \nabla_{q}\right) j^{0}\right| \leq\left|\partial_{t} j^{0}\right|+c\left|\nabla_{q} j^{0}\right|$, and the functions $|J|,\left|\partial_{t} j^{0}\right|$, and $c\left|\nabla_{q} j^{0}\right|$ are continuous and therefore bounded on the compact set $[0, T] \times \overline{B_{r+c T}}$. Hence the integrals in (15) and (16) are finite. This implies existence for all positive times. For negative times apply the same argument to the time-reversed current $\bar{\jmath}$, for which the same velocity bound holds.

Applying Corollary 1 to Bohm-Dirac theory, we obtain global existence of BohmDirac trajectories under very general conditions.

Theorem 2 Let $V \in C^{\infty}\left(\mathbb{R}^{3 N}, \operatorname{Herm}\left(\mathbb{C}^{4^{N}}\right)\right)$ and $\psi(t)=\mathrm{e}^{-\mathrm{i} t H_{\mathrm{D}}} \psi(0)$ with $\psi(0) \in$ $C^{\infty}\left(\mathbb{R}^{3 N}, \mathbb{C}^{4^{N}}\right) \cap L^{2}\left(\mathbb{R}^{3 N}, \mathbb{C}^{4^{N}}\right)$ and $\|\psi(0)\|=1$.

Then the solution $Q_{q}(t)=\left(\boldsymbol{Q}_{1}(t), \ldots, \boldsymbol{Q}_{N}(t)\right)$ of (15) with $Q_{q}(0)=q$ exists globally in time for almost all $q \in \mathbb{R}^{3 N}$ relative to the measure $\mu_{0}(d q)=|\psi(0, q)|^{2} d q$, and the $|\psi(t)|^{2}$ distributions are equivariant. 
Proof. According to $\left[8\right.$, for $\psi(0) \in C_{0}^{\infty}\left(\mathbb{R}^{3 N}, \mathbb{C}^{4^{N}}\right)$ one has $\psi(t) \in C_{0}^{\infty}\left(\mathbb{R}^{3 N}, \mathbb{C}^{4^{N}}\right)$ and $\psi(t, q) \in C^{\infty}\left(\mathbb{R} \times \mathbb{R}^{3 N}, \mathbb{C}^{4^{N}}\right)$. But then linearity and the finite propagation speed (Proposition 1.1 in [8]) imply that $\psi(t, q) \in C^{\infty}\left(\mathbb{R} \times \mathbb{R}^{3 N}, \mathbb{C}^{4^{N}}\right)$ also for $\psi(0) \in$ $C^{\infty}\left(\mathbb{R}^{3 N}, \mathbb{C}^{4^{N}}\right) \cap L^{2}\left(\mathbb{R}^{3 N}, \mathbb{C}^{4^{N}}\right)$. Hence, the Dirac current (10) satisfies (11). Since $|J| \leq c \sqrt{N} j^{0}$, Corollary 1 implies the theorem.

Corollary 2 Let now $\mathcal{Q}=\mathbb{R}^{d} \backslash \cup_{\ell=1}^{m} S_{\ell}$, where $S_{\ell}$ is a hyperplane with codimension $\geq 2$ for $\ell=1, \ldots, m$, and let $j=\left(j^{0}, J\right)$ be a current as defined in (11). Suppose that there is a global bound $c$ on velocities, $|J| \leq c j^{0}$, and that $J$ and the first order derivatives of $j^{0}$ are bounded on bounded sets.

Then for $\mu_{0}$-almost all $q \in \mathbb{R}^{d}$, the solution of (8) starting at $Q(0)=q$ exists for all times, and the family of measures $\mu_{t}$ is equivariant.

Proof. First note that $\mathbb{R}^{d} \backslash \mathcal{Q}$ is a Lebesgue-null set and hence also a $\mu_{0}$-null set. For $q \in \mathcal{Q}$ we apply Theorem 11. The conditions (15) and (16) of Theorem 1 follow as in the proof of Corollary 1 using the fact that $J$ and the derivatives of $j^{0}$ are locally bounded.

To check (17), let $d_{\ell}$ be the dimension of $S_{\ell}$ and assume without loss of generality that $S_{\ell}$ contains the origin. Then with $|J| \leq C$ on $B_{r+c T}^{d}$, the ball of radius $r+c T$ around the origin in $\mathbb{R}^{d}$, and $B_{r+c T}^{d} \subset B_{r+c T}^{d_{\ell}} \times B_{r+c T}^{d-d_{\ell}}$ we find that

$$
\int_{0}^{T} d t \int_{B_{r+c T}^{d}} d q \frac{|J(t, q)|}{\operatorname{dist}\left(q, S_{\ell}\right)} \leq T \int_{\substack{B_{r+c T}^{d_{\ell}} \\ B_{r+c T}^{d-d_{\ell}}}} d y \frac{C}{|y|}<\infty .
$$

\section{Global Existence of Bohmian Mechanics}

We now apply Theorem 1 to Bohmian mechanics and consider the abstract Hamiltonian

$$
H_{0}=-\frac{1}{2}\left(\mathfrak{m}^{-\frac{1}{2}}\left(\nabla_{q}-\mathrm{i} A(q)\right)\right)^{2} \mathbf{1}_{\mathbb{C}^{k}}+V(q), \quad D\left(H_{0}\right)=C_{0}^{\infty}\left(\mathcal{Q}, \mathbb{C}^{k}\right),
$$

where, for the moment, $A \in H_{\mathrm{loc}}^{1}\left(\mathbb{R}^{d}, \mathbb{R}^{d}\right)$ and $V \in L_{\mathrm{loc}}^{2}\left(\mathcal{Q}, \operatorname{Herm}\left(\mathbb{C}^{k}\right)\right)$. The mass matrix $\mathfrak{m}=\operatorname{diag}\left(m_{1}, \ldots, m_{d}\right)$ has positive entries $m_{i}>0$. These conditions assure that $H_{0}$ is well defined and symmetric on $C_{0}^{\infty}\left(\mathcal{Q}, \mathbb{C}^{k}\right)$. Since $H_{0}$ commutes with complex conjugation, $H_{0}$ has at least on self-adjoint extension. We also assume that $\mathcal{Q}=\mathbb{R}^{d} \backslash$ $\cup_{\ell=1}^{m} S_{\ell}$ where each $S_{\ell}$ is a $(d-3)$-dimensional hyperplane in $\mathbb{R}^{d}$. As to be explained in the example below, for $d=3 N$ the coincidence set of $N$ particles moving in $\mathbb{R}^{3}$ has exactly this structure and therefore singular pair-potentials like the Coulomb potential are included. In these abstract terms the Bohmian equation of motion reads

$$
\frac{d Q}{d t}(t)=\mathfrak{m}^{-1} \operatorname{Im} \frac{\psi^{*}\left(\nabla_{q}-\mathrm{i} A\right) \psi}{\psi^{*} \psi}(t, Q(t)) .
$$


Theorem 3 Let $H$ be a self-adjoint extension of $H_{0}$ as in (22) with domain $D(H)$. Suppose that for some $\psi(0) \in D(H)$ with $\|\psi(0)\|=1$ the solution $\psi(t)=\mathrm{e}^{-\mathrm{i} t H} \psi(0)$ of the Schrödinger equation satisfies

(i) $\psi \in C^{2}\left(\mathbb{R} \times \mathcal{Q}, \mathbb{C}^{k}\right)$,

(ii) for every $T>0$ there is a constant $C_{T}<\infty$ such that

$$
\int_{-T}^{T} d t\left(\||\nabla \psi(t)|\|^{2}+\||A \psi(t)|\|^{2}+\|A \cdot \nabla \psi(t)\|^{2}\right)<C_{T} .
$$

Then the solution $Q_{q}(t)$ of (23) with $Q_{q}(0)=q$ exists globally in time for almost all $q \in \mathbb{R}^{d}$ relative to the measure $\mu_{0}(d q)=|\psi(0, q)|^{2} d q$, and the $|\psi(t)|^{2}$ distributions are equivariant.

\section{Remark.}

9. Note that condition (i) in Theorem 3 is typically satisfied only if the potentials $A$ and $V$ are sufficiently smooth on $\mathcal{Q}$, more than we required after (22). We decided to state the condition in terms of $\psi$ since the exact type of smoothness required for $A$ and $V$ depends on, among other factors, the dimension $d$.

Proof of Theorem [3. First note that $\mathbb{R}^{d} \backslash \mathcal{Q}$ is a Lebesgue-null set and hence also a $\mu_{0}$-null set. For $q \in \mathcal{Q}$ we apply Theorem 1]. According to Section 2.3] and by virtue of (i), the Schrödinger current

$$
j(t, q)=\left(\psi^{*}(t, q) \psi(t, q), \mathfrak{m}^{-1} \operatorname{Im} \psi^{*}(t, q)\left(\nabla_{q}-\mathrm{i} A(q)\right) \psi(t, q)\right)
$$

satisfies (11). We now check (15), (16) and (17), in order to prove existence for positive times. For negative times one concludes analogously by applying exactly the same arguments to the time reversed current.

With $\psi(t)=\mathrm{e}^{-\mathrm{i} t H} \psi(0)$, the Cauchy-Schwarz inequality, and (ii) we obtain

$$
\begin{aligned}
\int_{\mathcal{Q}} d q\left|\partial_{t} j^{0}(t, q)\right| & =\int_{\mathbb{R}^{d}} d q\left|\partial_{t} \psi^{*}(t, q) \psi(t, q)\right| \leq 2 \int_{\mathbb{R}^{d}} d q\left|\psi^{*}(t, q) H \psi(t, q)\right| \\
& \leq 2\|H \psi(t)\|=2\|H \psi(0)\| .
\end{aligned}
$$

For the second term in (15) we find, after a straightforward computation involving Cauchy-Schwarz first on $\mathbb{C}^{k}$ and then on $L^{2}\left(\mathbb{R}^{d}\right)$ and finally on $L^{2}([0, T])$, that

$$
\begin{aligned}
\int_{0}^{T} d t \int_{\mathcal{Q} \backslash \mathscr{N}_{t}} d q\left|\frac{J}{j^{0}} \cdot \nabla j^{0}(t, q)\right| & \leq \frac{1}{m_{0}} \int_{0}^{T} d t \int_{\mathbb{R}^{d}} d q\left(|\nabla \psi(t, q)|^{2}+|\psi(t, q)||A(q) \cdot \nabla \psi(t, q)|\right) \\
& \leq \frac{C_{T}+\sqrt{T C_{T}}}{m_{0}},
\end{aligned}
$$

where $m_{0}=\min \left\{m_{1}, \ldots, m_{d}\right\}$. Hence, (15) holds. Analogously (16) follows from

$$
\int_{0}^{T} d t \int_{\mathcal{Q}} d q|J(t, q)| \leq \int_{0}^{T} d t \frac{1}{m_{0}} \int_{\mathbb{R}^{d}} d q|\psi(t, q)|(|\nabla \psi(t, q)|+|A(q) \psi(t, q)|) \leq \frac{\sqrt{T C_{T}}}{m_{0}} .
$$


We now come to (17). Since $S_{\ell}$ is a $(d-3)$-dimensional hyperplane, it can be written as $S_{\ell}=\left\{q \in \mathbb{R}^{d}: y_{\ell}(q)=a_{\ell}\right\}$ with $y_{\ell}: \mathbb{R}^{d} \rightarrow \mathbb{R}^{3}, q \mapsto\left(q \cdot y_{\ell}^{1}, q \cdot y_{\ell}^{2}, q \cdot y_{\ell}^{3}\right)$ where $y_{\ell}^{1}, y_{\ell}^{2}$, $y_{\ell}^{3}$ are 3 orthogonal unit vectors normal to the hyperplane $S_{\ell}$ and $a_{\ell} \in \mathbb{R}^{3}$ a constant. The distance to the hyperplane is given by $\operatorname{dist}\left(q, S_{\ell}\right)=\left|y_{\ell}(q)-a_{\ell}\right|$.

To prove (17) for $\delta=\infty$, we use the generalized Hardy inequality introduced in [4], Equation (25). It states that for all $\phi \in H^{1}\left(\mathbb{R}^{d}, \mathbb{C}\right)$, the first Sobolev space,

$$
\int_{\mathbb{R}^{d}} d q \frac{|\phi(q)|^{2}}{4\left|y_{\ell}(q)-a_{\ell}\right|^{2}} \leq \int_{\mathbb{R}^{d}} d q|\nabla \phi(q)|^{2} .
$$

Hence,

$$
\begin{aligned}
\int_{0}^{T} d t & \int_{\mathcal{Q}} d q \frac{\left|J(t, q) \cdot e_{\ell}(q)\right|}{\operatorname{dist}\left(q, S_{\ell}\right)} \leq \frac{1}{m_{0}} \int_{0}^{T} d t \int_{\mathbb{R}^{d}} d q \frac{\left|\psi^{*}(t, q)(\nabla-\mathrm{i} A(q)) \psi(t, q)\right|}{\left|y_{\ell}(q)-a_{\ell}\right|} \\
& \leq \frac{1}{m_{0}} \int_{0}^{T} d t \int_{\mathbb{R}^{d}} d q \frac{|\psi(t, q)|(|\nabla \psi(t, q)|+|A(q) \psi(t, q)|)}{\left|y_{\ell}(q)-a_{\ell}\right|} \\
& \leq \frac{1}{m_{0}} \int_{0}^{T} d t\left(\int_{\mathbb{R}^{d}} d q \frac{|\psi(t, q)|^{2}}{\left|y_{\ell}(q)-a_{\ell}\right|^{2}}\right)^{\frac{1}{2}}(\||\nabla \psi(t)|\|+\||A \psi(t)|\|) \\
& \leq \frac{1}{m_{0}} \int_{0}^{T} d t\left(2\||\nabla \psi(t)|\|^{2}+\||\nabla \psi(t)|\|\||A \psi(t)|\|\right) \leq \frac{3 C_{T}}{m_{0}} .
\end{aligned}
$$

We shall not try to verify the assumptions of Theorem 3 under as general as possible conditions on $A$ and $V$. Instead we consider two examples where they can be checked without too much effort.

Our first example concerns a molecular system in external fields. More precisely we consider $N$ electrons in $\mathbb{R}^{3}$ with configuration $q=\left(\boldsymbol{q}_{1}, \ldots, \boldsymbol{q}_{N}\right) \in \mathbb{R}^{3 N}$ interacting through Coulomb potentials

$$
V_{\mathrm{el}}(q)=\sum_{i=1}^{N-1} \sum_{j=i+1}^{N} \frac{1}{\left|\boldsymbol{q}_{i}-\boldsymbol{q}_{j}\right|}
$$

in the electric potential

$$
V_{\mathrm{nu}}(q)=-\sum_{i=1}^{N} \sum_{j=1}^{M} \frac{Z_{j}}{\left|\boldsymbol{q}_{i}-\boldsymbol{z}_{j}\right|}
$$

of $M$ static nuclei located at $\boldsymbol{z}_{j} \in \mathbb{R}^{3}$ with charges $Z_{j}, j=1, \ldots, M$. Furthermore we allow for an external magnetic field $\boldsymbol{B}(\boldsymbol{x})=\nabla \times \boldsymbol{A}(\boldsymbol{x})$ with $\boldsymbol{A} \in C^{\infty}\left(\mathbb{R}^{3}, \mathbb{R}^{3}\right)$ such that $\nabla \cdot \boldsymbol{A}=0$ and $\boldsymbol{B}$ and $\boldsymbol{A}$ are bounded. The Hamiltonian of the system thus is

$$
H_{\mathrm{mol}}=\left(-\frac{1}{2} \sum_{i=1}^{N}\left(\nabla_{\boldsymbol{q}_{i}}+\mathrm{i} \boldsymbol{A}\left(\boldsymbol{q}_{i}\right)\right)^{2}+V_{\mathrm{el}}(q)+V_{\mathrm{nu}}(q)\right) \mathbf{1}_{\left(\mathbb{C}^{2}\right) \otimes N}-\sum_{i=1}^{N} \boldsymbol{B}\left(\boldsymbol{q}_{i}\right) \cdot \boldsymbol{\sigma}_{i}
$$


with domain $D\left(H_{\mathrm{mol}}\right)=H^{2}\left(\mathbb{R}^{3 N},\left(\mathbb{C}^{2}\right)^{\otimes N}\right)$. Here $\boldsymbol{\sigma}_{i}$ is the vector of Pauli matrices acting on the spin index of particle $i$. It is well known that $V_{\mathrm{el}}, V_{\mathrm{nu}}$, and $\nabla_{q}$ are infinitesimally bounded with respect to $\Delta_{q}$. Hence $H_{\mathrm{mol}}=-\frac{1}{2} \Delta_{q}+R$ with

$$
R:=\left(-\frac{1}{2} \sum_{i=1}^{N}\left(2 \mathrm{i} \boldsymbol{A}\left(\boldsymbol{q}_{i}\right) \cdot \nabla_{\boldsymbol{q}_{i}}-\boldsymbol{A}\left(\boldsymbol{q}_{i}\right)^{2}\right)+V_{\mathrm{el}}(q)+V_{\mathrm{nu}}(q)\right) \mathbf{1}_{\left(\mathbb{C}^{2}\right) \otimes N}-\sum_{i=1}^{N} \boldsymbol{B}\left(\boldsymbol{q}_{i}\right) \cdot \boldsymbol{\sigma}_{i}
$$

is self-adjoint by virtue of Kato's theorem.

Corollary 3 Let $\psi(t)=\mathrm{e}^{-\mathrm{i} t H_{\mathrm{mol}}} \psi(0)$ with $\psi(0) \in C^{\infty}\left(H_{\mathrm{mol}}\right)=\cap_{n=1}^{\infty} D\left(\left(H_{\mathrm{mol}}\right)^{n}\right)$ and $\|\psi(0)\|=1$. Then the Bohmian trajectories $Q_{q}(t)$ exist globally in time for almost all $q \in \mathbb{R}^{3 N}$ relative to the measure $|\psi(0, q)|^{2} d q$, and the $|\psi(t)|^{2}$ distributions are equivariant.

Proof. First note that $H_{\mathrm{mol}}$ is of the form (22) with $d=3 N$ and $k=2^{N}$. The configuration space of the system is

$$
\mathcal{Q}=\mathbb{R}^{3 N} \backslash\left(\left(\cup_{i=1}^{N-1} \cup_{j=i}^{N}\left\{q \in \mathbb{R}^{3 N}: \boldsymbol{q}_{i}=\boldsymbol{q}_{j}\right\}\right) \cup\left(\cup_{i=1}^{N} \cup_{j=1}^{M}\left\{q \in \mathbb{R}^{3 N}: \boldsymbol{q}_{i}=\boldsymbol{z}_{j}\right\}\right)\right),
$$

where the $N(N-1) / 2$ electron-electron and the $N M$ electron-nucleus coincidence hyperplanes are all $(3 N-3)$-dimensional. As remarked above, $H_{\text {mol }}$ is self-adjoint on $H^{2}\left(\mathbb{R}^{3 N},\left(\mathbb{C}^{2}\right)^{\otimes N}\right)$ and thus satisfies the hypotheses of Theorem 3 . Hence it suffices to check that $\psi(t)$ satisfies the hypotheses (i) and (ii) of Theorem 3 . As for (i), note that all potentials in (24) are $C^{\infty}$ on $\mathcal{Q}$. Then methods of elliptic regularity can be applied to show that for $\psi(0) \in C^{\infty}\left(H_{\text {mol }}\right)$ the solution of the Schrödinger equation satisfies $\psi \in C^{\infty}(\mathbb{R} \times \mathcal{Q})$. For details see the appendix in [4]. Finally notice that, since $\boldsymbol{A}$ is assumed to be bounded and since $\|\psi(t)\|=\|\psi(0)\|$, (ii) follows if we can show that the kinetic energy $\||\nabla \psi(t)|\|$ remains bounded. This is also standard but we give the short argument anyway: since $R$ is infinitesimally bounded with respect to $\Delta$, there are constants $0<a<1$ and $b>0$ such that $\|R \phi\| \leq a\left\|\frac{1}{2} \Delta \phi\right\|+b\|\phi\|$ for all $\phi \in H^{2}=D\left(H_{\text {mol }}\right)$. Hence

$$
\begin{aligned}
\|\Delta \psi(t)\| & =2\left\|\left(\frac{1}{2} \Delta-R+R\right) \psi(t)\right\| \leq 2\|H \psi(t)\|+2\|R \psi(t)\| \\
& \leq 2\|H \psi(t)\|+a\|\Delta \psi(t)\|+2 b\|\psi(t)\|
\end{aligned}
$$

together with $\|H \psi(t)\|=\|H \psi(0)\|$ and $\|\psi(t)\|=\|\psi(0)\|$ implies

$$
\|\Delta \psi(t)\| \leq \frac{2\|H \psi(0)\|+2 b\|\psi(0)\|}{1-a}=C .
$$

But then also

$$
\||\nabla \psi(t)|\|^{2}=\langle\nabla \psi(t), \cdot \nabla \psi(t)\rangle=-\langle\psi(t), \Delta \psi(t)\rangle \leq\|\psi(t)\|\|\Delta \psi(t)\| \leq\|\psi(0)\| C .
$$

The last corollary coincides exactly with the result of [4] (see their Corollary 3.2). 
Corollary 4 In (22) let $k=1, A=0$ and $V=V_{1}+V_{2} \in C^{\infty}(\mathcal{Q}, \mathbb{C})$, where $V_{1}$ is bounded below and $V_{2}$ is $-\frac{1}{2} \Delta$-form bounded with relative bound $<1$. Then the form sum $H=-\frac{1}{2} \Delta+V$ is a self-adjoint extension of $H_{0}$ and for $\psi(t)=\mathrm{e}^{-\mathrm{i} t H} \psi(0)$ with $\psi(0) \in C^{\infty}(H)=\cap_{n=1}^{\infty} D\left(H^{n}\right),\|\psi(0)\|=1$, the Bohmian trajectories $Q_{q}(t)$ exist globally in time for almost all $q \in \mathbb{R}^{d}$ relative to the measure $|\psi(0, q)|^{2} d q$, and the $|\psi(t)|^{2}$ distributions are equivariant.

Proof. For the statement about the form sum see [12]. Again, as shown in the appendix of [4], elliptic regularity implies that $\psi \in C^{\infty}(\mathbb{R} \times \mathcal{Q})$. Hence, in order to apply Theorem 3 it suffices to show that that $\||\nabla \psi(t)|\|$ remains bounded. This follows by an argument analogous to the one given in the proof of Corollary 3. For the details see the proof of Corollary 3.2 in [4].

Acknowledgements: We thank Sheldon Goldstein for helpful remarks and Florian Theil for pointing out to us reference 9]. R.T. thanks the Mathematics Institute of the University of Warwick for hospitality and INFN for financial support.

\section{References}

[1] Bell, J. S.: "On the problem of hidden variables in quantum mechanics," Rev. Mod. Phys. 38, 447-452 (1966). Reprinted in Bell, J. S.: Speakable and unspeakable in quantum mechanics. Cambridge: Cambridge University Press (1987), p. 1.

[2] Berndl, K.: Zur Existenz der Dynamik in Bohmschen Systemen. Ph. D. thesis, Ludwig-Maximilians-Universität München. Aachen: Mainz Verlag (1995)

[3] Berndl, K., Daumer, M., Dürr, D., Goldstein, S., Zanghì, N.: "A Survey of Bohmian Mechanics," Il Nuovo Cimento 110B, 737-750 (1995), and quant-ph/9504010

[4] Berndl, K., Dürr, D., Goldstein, S., Peruzzi, G., Zanghì, N.: "On the global existence of Bohmian mechanics," Commun. Math. Phys. 173, 647-673 (1995), and quant-ph/9503013

[5] Bohm, D.: "A Suggested Interpretation of the Quantum Theory in Terms of "Hidden" Variables, I," Phys. Rev. 85, 166-179 (1952). Bohm, D.: "A Suggested Interpretation of the Quantum Theory in Terms of "Hidden" Variables, II," Phys. Rev. 85, 180-193 (1952)

[6] Bohm, D.: "Comments on an Article of Takabayasi concerning the Formulation of Quantum Mechanics with Classical Pictures," Progr. Theoret. Phys. 9, 273-287 (1953)

[7] Bohm, D., Hiley, B. J.: The Undivided Universe: An Ontological Interpretation of Quantum Theory. London: Routledge (1993) 
[8] Chernoff, P. R.: "Essential Self-Adjointness of Powers of Generators of Hyperbolic Equations," J. Funct. Anal. 12, 401-414 (1973)

[9] DiPerna, R. J., Lions, P. L.: "Ordinary differential equations, transport theory and Sobolev spaces," Invent. Math. 98, 511-547 (1989)

[10] Dürr, D.: Bohmsche Mechanik als Grundlage der Quantenmechanik. Berlin: Springer-Verlag (2001)

[11] Dürr, D., Goldstein, S., Zanghì, N.: "Quantum Equilibrium and the Origin of Absolute Uncertainty," J. Statist. Phys. 67, 843-907 (1992), and quant-ph/0308039

[12] Faris, W. G.: Self-adjoint operators. Lecture Notes in Mathematics 433. Berlin: Springer-Verlag (1975).

[13] Georgii, H.-O., Tumulka, R.: "Global Existence of Bell's Time-Inhomogeneous Jump Process for Lattice Quantum Field Theory," to appear in Markov Proc. Rel. Fields (2004); math.PR/0312294 and mp_arc 04-11

[14] Georgii, H.-O., Tumulka, R.: "Some Jump Processes in Quantum Field Theory," to appear in the proceedings of the DFG Priority Program "Interacting Stochastic Systems of High Complexity", Springer-Verlag (2004), and math.PR/0312326

[15] Holland, P. R.: The Quantum Theory of Motion. Cambridge: Cambridge University Press (1993)

[16] Lefschetz, S.: Differential Equations: Geometric Theory. New York, London: Interscience Publishers (1957) 